\title{
SEARCH FOR A CORRELATION BETWEEN VERY-HIGH-ENERGY GAMMA RAYS AND GIANT RADIO PULSES IN THE CRAB PULSAR
}

\section{E. Aliu ${ }^{1}$, S. Archambault 2 , T. Arlen ${ }^{3}$, T. Aune ${ }^{4}$, M. Beilicke $^{5}$, W. Benbow ${ }^{6}$, A. Bouvier ${ }^{4}$, J. H. Buckley ${ }^{5}$, V. BugaeV $^{5}$,} K. Byrum ${ }^{7}$, A. Cesarini ${ }^{8}$, L. Ciupik ${ }^{9}$, E. Collins-Hughes ${ }^{10}$, M. P. Connolly ${ }^{8}$, W. Cui ${ }^{11}$, R. DiCKherber $^{5}$, C. Duke $^{12}$,

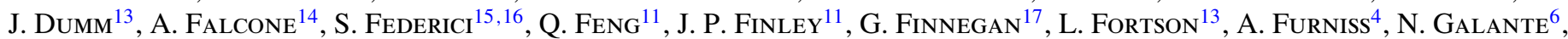
D. Gall ${ }^{18}$, G. H. Gillanders ${ }^{8}$, S. Godambe ${ }^{17}$, S. Griffin ${ }^{2}$, J. Grube ${ }^{9}$, G. Gyuk ${ }^{9}$, D. Hanna ${ }^{2}$, J. Holder ${ }^{19}$, H. Huan ${ }^{20}$, G. Hughes ${ }^{15}$, T. B. Humensky ${ }^{21}$, P. KaAret ${ }^{18}$, N. Karlsson ${ }^{13}$, Y. Khassen ${ }^{10}$, D. Kieda ${ }^{17}$, H. KrawcZynski ${ }^{5}$, F. KrennRich $^{22}$,

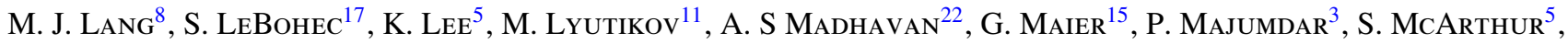
A. McCann ${ }^{23}$, P. Moriarty ${ }^{24}$, R. MukherjeEe ${ }^{1}$, T. Nelson ${ }^{13}$, A. O’Faoláin de Bhróithe ${ }^{10}$, R. A. ONG ${ }^{3}$, M. OrR ${ }^{22}$, A. N. Otte ${ }^{25}$, N. Park ${ }^{20}$, J. S. Perkins ${ }^{26,27}$, M. Pohl ${ }^{15,16}$, H. ProkOPH ${ }^{15}$, J. QuinN ${ }^{10}$, K. RAGAN ${ }^{2}$, L. C. Reyes ${ }^{28}$, P. T. Reynolds ${ }^{29}$, E. Roache ${ }^{6}$, D. B. SAXon ${ }^{19}$, M. Schroedter ${ }^{6}$, G. H. Sembroski ${ }^{11}$, G. D. Şentürk ${ }^{21}$, A. W. Smith ${ }^{17}$, D. StaszaK ${ }^{2}$, I. Telezhinsky ${ }^{15,16}$, G. Tešić ${ }^{2}$, M. Theiling ${ }^{11}$, S. Thibadeau ${ }^{5}$, K. Tsurusaki $^{18}$, A. Varlotta ${ }^{11}$, S. Vincent $^{15}$,

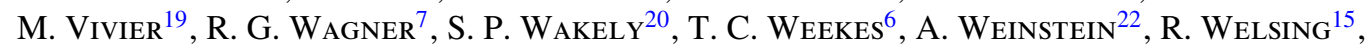
D. A. Williams ${ }^{4}$, B. ZitZer ${ }^{7}$, AND V. Kondratiev ${ }^{30,31}$

${ }^{1}$ Department of Physics and Astronomy, Barnard College, Columbia University, NY 10027, USA

${ }^{2}$ Physics Department, McGill University, Montreal, QC H3A 2T8, Canada

${ }^{3}$ Department of Physics and Astronomy, University of California, Los Angeles, CA 90095, USA

${ }^{4}$ Santa Cruz Institute for Particle Physics and Department of Physics, University of California, Santa Cruz, CA 95064, USA ${ }^{5}$ Department of Physics, Washington University, St. Louis, MO 63130, USA

${ }^{6}$ Fred Lawrence Whipple Observatory, Harvard-Smithsonian Center for Astrophysics, Amado, AZ 85645, USA; schroedter@ veritas.sao.arizona.edu ${ }_{7}$ Argonne National Laboratory, 9700 S. Cass Avenue, Argonne, IL 60439, USA

${ }^{8}$ School of Physics, National University of Ireland Galway, University Road, Galway, Ireland

${ }_{9}^{9}$ Astronomy Department, Adler Planetarium and Astronomy Museum, Chicago, IL 60605, USA

${ }^{10}$ School of Physics, University College Dublin, Belfield, Dublin 4, Ireland

${ }^{11}$ Department of Physics, Purdue University, West Lafayette, IN 47907, USA

${ }^{12}$ Department of Physics, Grinnell College, Grinnell, IA 50112-1690, USA

${ }^{13}$ School of Physics and Astronomy, University of Minnesota, Minneapolis, MN 55455, USA

${ }^{14}$ Department of Astronomy and Astrophysics, 525 Davey Lab, Pennsylvania State University, University Park, PA 16802, USA

${ }^{15}$ DESY, Platanenallee 6, 15738 Zeuthen, Germany

${ }^{16}$ Institute of Physics and Astronomy, University of Potsdam, 14476 Potsdam-Golm, Germany

${ }^{17}$ Department of Physics and Astronomy, University of Utah, Salt Lake City, UT 84112, USA

18 Department of Physics and Astronomy, University of Iowa, Van Allen Hall, Iowa City, IA 52242, USA

${ }^{19}$ Department of Physics and Astronomy and the Bartol Research Institute, University of Delaware, Newark, DE 19716, USA

${ }^{20}$ Enrico Fermi Institute, University of Chicago, Chicago, IL 60637, USA

${ }^{21}$ Physics Department, Columbia University, New York, NY 10027, USA

22 Department of Physics and Astronomy, Iowa State University, Ames, IA 50011, USA

${ }^{23}$ Kavli Institute for Cosmological Physics, University of Chicago, Chicago, IL 60637, USA; mccann@kicp.uchicago.edu

${ }^{24}$ Department of Life and Physical Sciences, Galway-Mayo Institute of Technology, Dublin Road, Galway, Ireland

${ }^{25}$ School of Physics and Center for Relativistic Astrophysics, Georgia Institute of Technology, 837 State Street NW, Atlanta, GA 30332-0430, USA; nepomuk.otte@gmail.com

${ }^{26}$ CRESST and Astroparticle Physics Laboratory NASA/GSFC, Greenbelt, MD 20771, USA

${ }^{27}$ University of Maryland, Baltimore County, 1000 Hilltop Circle, Baltimore, MD 21250, USA

${ }^{28}$ Physics Department, California Polytechnic State University, San Luis Obispo, CA 94307, USA

${ }^{29}$ Department of Applied Physics and Instrumentation, Cork Institute of Technology, Bishopstown, Cork, Ireland

${ }^{30}$ ASTRON, The Netherlands Institute for Radio Astronomy, Postbus 2, 7990 AA, Dwingeloo, The Netherlands

${ }^{31}$ Astro Space Center of the Lebedev Physical Institute, Profsoyuznaya str. 84/32, Moscow 117997, Russia Received 2012 July 2; accepted 2012 October 12; published 2012 November 16

\begin{abstract}
We present the results of a joint observational campaign between the Green Bank radio telescope and the VERITAS gamma-ray telescope, which searched for a correlation between the emission of very-high-energy (VHE) gamma rays $\left(E_{\gamma}>150 \mathrm{GeV}\right)$ and giant radio pulses (GRPs) from the Crab pulsar at $8.9 \mathrm{GHz}$. A total of 15,366 GRPs were recorded during $11.6 \mathrm{hr}$ of simultaneous observations, which were made across four nights in 2008 December and in 2009 November and December. We searched for an enhancement of the pulsed gamma-ray emission within time windows placed around the arrival time of the GRP events. In total, eight different time windows with durations ranging from $0.033 \mathrm{~ms}$ to $72 \mathrm{~s}$ were positioned at three different locations relative to the GRP to search for enhanced gamma-ray emission which lagged, led, or was concurrent with, the GRP event. Furthermore, we performed separate searches on main pulse GRPs and interpulse GRPs and on the most energetic GRPs in our data sample. No significant enhancement of pulsed VHE emission was found in any of the preformed searches. We set upper limits of 5-10 times the average VHE flux of the Crab pulsar on the flux simultaneous with interpulse GRPs on single-rotation-period timescales. On $\sim 8 \mathrm{~s}$ timescales around interpulse GRPs, we set an upper limit of
\end{abstract}


2-3 times the average VHE flux. Within the framework of recent models for pulsed VHE emission from the Crab pulsar, the expected VHE-GRP emission correlations are below the derived limits.

Key words: gamma rays: stars - pulsars: individual: B0531+21

Online-only material: color figures

\section{INTRODUCTION}

The Crab pulsar, PSR B0531+21, is a powerful young pulsar and one of the most studied objects in the sky. It is one of the brightest pulsars in the high-energy gamma-ray regime (Fierro et al. 1998; Abdo et al. 2010) and the only pulsar so far to be detected above $100 \mathrm{GeV}$ (Aliu et al. 2011; Aleksić et al. 2012). The Crab pulsar is also one of only several known pulsars which exhibit the giant radio pulse phenomenon (Knight 2006): single radio pulses with flux densities that greatly exceed the average pulse flux density and can, at their maximum, be as bright as a few million Janskys (Soglasnov 2007). The energies of giant pulses follow a power-law distribution (Cordes et al. 2004; Popov \& Stappers 2007) in contrast to the regular pulses that obey a Gaussian or log-normal distribution (Burke-Spolaor et al. 2012). High-resolution time measurements of individual giant radio pulses (GRPs) reveal pulses which can be a few microseconds to a few nanoseconds wide (Hankins et al. 2003), with the narrower GRPs possessing the highest flux density.

The pulse profile of the Crab pulsar is dominated across the electromagnetic spectrum, from radio waves to very-highenergy (VHE) gamma rays, by two emission peaks, referred to as the main pulse and interpulse. These peaks occur at phases 0.0 and 0.4 , respectively, with a "bridge" of enhanced emission appearing between these two peaks in the optical, X-ray, and gamma-ray pulse profiles (Oosterbroek et al. 2008; Mineo et al. 2006; Kuiper et al. 2001; Abdo et al. 2010). Above $100 \mathrm{GeV}$, significant emission is only observed during the main and interpulse (Aliu et al. 2011; Aleksić et al. 2012). In the radio-pulse profile, several different components appear at different frequencies, including a precursor to the main radio pulse and two "high frequency components" (HFCs), which only appear above $\sim 5 \mathrm{GHz}$ and occur at phases $\sim 0.77$ and 0.93 (Moffett \& Hankins 1996). In the Crab pulsar, giant radio pulses have been observed to occur during both the main pulse and the interpulse. Indeed, it has been suggested that the Crab radio profile is composed of entirely GRPs, with the "regular" pulsar pulse corresponding to the main pulse precursor component (Popov et al. 2006). The case for the regular pulse is difficult to disentangle, since single regular pulses cannot be observed over the nebular background. Stark differences in the characteristics of main pulse GRPs and interpulse GRPs have been revealed by observations of the Crab pulsar between 6 and $10.5 \mathrm{GHz}$. In this frequency range, interpulse GRPs are typically several microseconds long and populate a set of regularly spaced frequency bands (Hankins \& Eilek 2007). On the other hand, main pulse GRPs exhibit broadband spectra and appear as a succession of narrow pulses ranging from unresolved widths below $0.4 \mathrm{~ns}$ to widths of a few microseconds (Hankins et al. 2003; Hankins \& Eilek 2007). These striking differences suggest that the emission mechanisms may differ between the main pulse GRPs and interpulse GRPs in the Crab pulsar above $5 \mathrm{GHz}$.

The mechanisms responsible for the generation of GRPs are still unknown. Changes in the coherence of the plasma beam, which is believed to be responsible for the normal pulsed radio emission, can in principle explain the generation of GRPs. However, mechanisms which primarily affect the coherence of the plasma have no effect on the incoherent emission from pulsars and, thus, no enhancement is expected in the gamma-ray emission in connection with GRP events. Mechanisms which increase the rate of particle production within the magnetospheric emission region, or which change the direction of the emission beam, should, however, affect the higher energy incoherent emission. Such mechanisms may create an enhancement in the gamma-ray emission from the pulsar.

The exceptional emission band structure seen in interpulse GRPs from the Crab pulsar at high frequencies reported by Hankins \& Eilek (2007) prompted a quantitative model for their generation by Lyutikov (2007). This model argues that GRPs are generated in a dense plasma region close to the last closed magnetic field line. Occasional magnetic field reconnections excite the plasma producing a particle beam with a high Lorentz factor which emits a GRP via anomalous cyclotron resonance. This model, when fit to the interpulse GRP data from Hankins $\&$ Eilek (2007), returns a predicted Lorentz factor for the beam, $\gamma=\mathcal{O}\left(10^{8}\right)$, which is large enough to cause the particles within the beam to generate curvature photons with energies as high as tens of GeV. Thus, a feature of this model is a prediction of an enhancement in the gamma-ray emission correlated with high-frequency interpulse GRPs from the Crab pulsar.

Several studies have been performed to examine a possible connection between GRPs in the Crab and higher energy incoherent emission. Lundgren et al. (1995) studied 50-220 keV gamma rays recorded by the OSSE instrument on board NASA's Compton Gamma-ray Observatory along side Green Bank radio data recorded at $800,812.5$, and $1330 \mathrm{MHz}$. No enhancement was seen in the gamma-ray emission on 2 minute timescales concurrent with GRP events. This study yielded an upper limit on the average gamma-ray flux from the pulsar over 2 minutes concurrent with GRPs of 2.5 times the average pulsed gamma-ray flux. Based on the non-detection of a gammaray enhancement, the authors argue in favor of coherence changes within the emission plasma as the source of GRP generation. Shearer et al. (2003), however, observed a significant $3 \%$ enhancement in the optical main pulse concurrent with the period of emission of GRPs measured at $1380 \mathrm{MHz}$. No enhancement was seen in the interpulse. This observation suggests a link between coherent radio emission and incoherent optical emission in the Crab pulsar. Small changes in the paircreation rate leading to localized density increases within the emission plasma could create the GRP event and provide a small enhancement in the optical incoherent emission.

Recently, Bilous et al. (2011) studied 0.1-5 GeV gamma rays recorded by the Fermi-LAT in conjunction with $8.9 \mathrm{GHz}$ observations of the Crab performed by the Green Bank radio telescope. No enhancement was seen in the gamma-ray flux within the main pulse, interpulse, and bridge, during single rotation periods which contained a GRP. This yielded a limit on the flux from the pulsar during rotation periods concurrent 
Table 1

Summary of the Radio and Gamma-ray Data Used in This Study

\begin{tabular}{lccccc}
\hline \hline $\begin{array}{l}\text { Date } \\
(\mathrm{MJD})\end{array}$ & No. of GRPs & $\begin{array}{c}\text { No. of GRPs } \\
\text { Overlap }\end{array}$ & $\begin{array}{c}\text { No. of } \gamma \text {-ray } \\
\text { Candidates }\end{array}$ & $\begin{array}{c}\text { T Overlap } \\
(\mathrm{min})\end{array}$ & $\begin{array}{c}\text { Center Freq. } \\
(\mathrm{MHz})\end{array}$ \\
\hline 54829 & 280 & 150 & 5566 & 138.365 & 8832 \\
55153 & 7813 & 5937 & 8738 & 200.232 & 8900 \\
55158 & 3771 & 3243 & 7928 & 182.881 & 8900 \\
55180 & 6916 & 6036 & 7861 & 180.107 & 8900 \\
\hline Total & 18780 & 15366 & 30093 & 701.585 & 3.2 \\
\hline
\end{tabular}

Notes. Measurements were made on four separate nights with a total $11.6 \mathrm{hr}$ of simultaneous data accumulated. The significantly smaller number of GRP events found on the first night of observations is likely due to higher attenuation of the radio input signal.

with GRPs of less than four times the average gamma-ray flux from the pulsar. This result suggests that enhanced pair creation is not the dominant factor in the emission of GRPs in the Crab and supports the idea that local coherence changes in the magnetospheric plasma cause GRPs, or, that the putative enhanced gamma-ray emission is beamed in a different direction than the radio emission and thus unobservable from Earth. Another recent study by Bilous et al. (2012), which examined 1.4-4.5 keV X-rays from Chandra observations of the Crab pulsar in conjunction with radio observations by the Green Bank telescope at $1.1-1.9 \mathrm{GHz}$, found no enhancement in the X-ray flux in connection with GRPs. This study yielded limits on the flux enhancement in the main pulse and interpulse concurrent with GRP events of $10 \%$ and $30 \%$, respectively.

Along with the aforementioned studies which searched for a correlation between GRPs and incoherent emission below $10 \mathrm{GeV}$, a GRP correlation search was performed on the Crab pulsar at TeV energies in the early 1970s (Argyle et al. 1974). This pioneering attempt was performed with the $25 \mathrm{~m}$ DRAO radio telescope and the Whipple gamma-ray telescope almost two decades before the first conclusive detection of a $\mathrm{TeV}$ gamma-ray source (Weekes et al. 1989). No TeV-GRP correlation was observed and we are aware of no other study on this subject with subsequent $\mathrm{TeV}$ instruments.

VERITAS is an array of four imaging atmospheric Cherenkov gamma-ray telescopes located at the Fred Lawrence Whipple Observatory at the base of Mount Hopkins in southern Arizona (Holder et al. 2006). Each telescope uses a 12 m Davies-Cotton reflector (Davies \& Cotton 1957) instrumented with a photomultiplier tube (PMT) camera with 499 pixels. The array is sensitive to gamma rays with energies between $100 \mathrm{GeV}$ and $30 \mathrm{TeV}$. The recent detection by VERITAS of pulsed emission above $100 \mathrm{GeV}$ from the Crab (Aliu et al. 2011) is not expected within the context of contemporary pulsar models in which curvature radiation is the dominant gamma-ray emission mechanism. The origin of the power-law extension of the spectral energy distribution above $100 \mathrm{GeV}$ seen now by both VERITAS and MAGIC (Aleksić et al. 2012) is poorly understood. A possible explanation is that the VHE emission is caused by inverse Compton (IC) upscattering of soft photons by particles accelerated in the outer magnetosphere (Romani 1996; Lyutikov et al. 2012; Du et al. 2012). Another possible explanation is that the pulsed VHE emission is produced when pulsed magnetospheric X-ray photons are upscattered by relativistic plasma in the wind outside the light cylinder (Aharonian et al. 2012). Given that the origin of the VHE emission and the generation of GRPs in the Crab pulsar are both poorly understood energetic phenomena, we are motivated to probe whether they are connected.

The remainder of this paper is structured in the following way. In Section 2 we describe the simultaneous observations of the Crab pulsar made with the GBT and VERITAS gammaray telescopes. We also describe the extraction of GRP events from the radio data set. In Section 3 we discuss the gamma-ray data processing and our strategy for searching for correlated emission within the radio and gamma-ray data sets. In Section 4 we describe a Monte Carlo simulation of the Crab pulsar signal within the VERITAS data set, which is used to calculate the strength of any correlation found in the data sets. In Section 5 we detail the results of the enhancement search and in Section 6 we provide some concluding remarks.

\section{OBSERVATIONS}

Simultaneous observations of the Crab pulsar were made by VERITAS and the $100 \mathrm{~m}$ Robert C. Byrd Green Bank Telescope (GBT) on 2008 December 29, 2009 November 18 and 23, and 2009 December 15. A total of $11.6 \mathrm{hr}$ of simultaneous data were recorded across these four nights with 2.3, 3.33, 3.04, and $3.00 \mathrm{hr}$ acquired, respectively. Table 1 gives a summary of the data used in this study.

\subsection{GBT Observations and GRP Selection}

The radio data presented here were acquired using the Green Bank Ultimate Pulsar Processor Instrument (GUPPI) in search mode. The total bandwidth of $800 \mathrm{MHz}$ was centered at 8832 or $8900 \mathrm{MHz}$ and split into 128 or 256 frequency channels for our observations in 2008 and 2009, respectively. Full Stokes parameters were recorded at a sampling interval of $40.96 \mu \mathrm{s}$ in 2008, whereas total intensity was recorded with a sampling interval of $3.2 \mu \mathrm{s}$ in our 2009 observations.

Recorded data from every session were dedispersed using the PRESTO pulsar software package, ${ }^{32}$ and searched for all singlepulse events with a peak signal-to-noise ratio $(\mathrm{S} / \mathrm{N})$ seven times greater than the average radio signal. The dedispersion was done using the contemporaneous dispersion measure (DM) value stated in the Jodrell Bank Crab pulsar monthly ephemeris ${ }^{33}$ (Lyne et al. 1993). The GRP selection was performed with the PRESTO tool singlepulse_search.py, which convolves the dedispersed time series with a series of boxcar functions of different widths. The times of arrival of the selected GRP events were converted into Tempo ${ }^{34}$ format and transformed to barycentric dynamical time (TDB) for the correlation analysis with the VERITAS events.

Between the observation sessions in 2008 and 2009, the DM has increased from $56.7883 \mathrm{pc} \mathrm{cm}^{-3}$ in 2008 December 15 to $56.8279 \mathrm{pc} \mathrm{cm}^{-3}$ in 2009 December. We estimate timing errors

\footnotetext{
32 http://www.cv.nrao.edu/ sransom/presto/

33 http://www.jb.man.ac.uk/pulsar/crab.html

$34 \mathrm{http}: / /$ tempo.sourceforge.net
} 
at our high observing frequency of $8.9 \mathrm{GHz}$ to be $\sim 0.4 \mu \mathrm{s}$, which is less than our sampling time. Here we assume a smooth variation in the DM and that the changes in the DM between our observing sessions are certainly less than the measured change of the $\mathrm{DM}$ of $\approx 0.04 \mathrm{pc} \mathrm{cm}^{-3}$ over the course of one year.

The system equivalent flux density is mostly determined by the Crab Nebula. Flux densities of the Crab Nebula were calculated with the relation $S(f)=955 \times(f / \mathrm{GHz})^{-0.27} \mathrm{Jy}$ (Cordes et al. 2004), accounting for the fact that at $8.9 \mathrm{GHz}$ the solid angle of the GBT beam covers only $6.25 \%$ of the area occupied by the nebula. We estimate the system equivalent flux density to be about 0.2 Jy for our 2008 observations, and about $0.7 \mathrm{Jy}$ for the observing session in 2009. The smaller number of GRPs found in the 2008 data set is likely due to a higher attenuation in the receiver system in 2008 where two back-end readouts were used together: the GBT Pulsar Spigot Card and the GBT Mk5 disk readout. The clumping of the barycentric arrival times of the GRPs clearly seen in the 2009 observations (see Figures 1 and 4) agrees with what one would expect from refractive interstellar scintillations (RISS). At $8.9 \mathrm{GHz}$, the characteristic timescale of RISS is about 80 minutes (Bilous et al. 2011).

\subsection{VERITAS Observations}

The VERITAS observations were made under the best possible sky conditions with each of the four telescopes fully operational. These observations were made with zenith angles smaller than $30^{\circ}$ and an average zenith angle of $16^{\circ}$, yielding the lowest possible energy threshold. All data were acquired in wobble mode (Fomin et al. 1994). This mode of observation places the object under study at an offset of 0.5 from the center of the field of view of the telescope, allowing for a simultaneous measurement of the background in other regions of the field of view with the same acceptance as the region containing the source.

Before an event is recorded with VERITAS, at least two of the four telescopes must trigger on a Cherenkov flash within $50 \mathrm{~ns}$. A single telescope trigger is formed when three or more adjacent PMTs in the camera register at least six photoelectrons within $9 \mathrm{~ns}$. When the trigger condition is satisfied, the PMT traces in each telescope are read out by 500 mega-sample per second flash analog-to-digital converters which are located at each telescope. For each event, the VERITAS data stream contains the GPS time stamp from each of the four telescopes along with the digitized PMT traces. In the entire VERITAS data set presented in this work, the four GPS time stamps never diverged by more than $10 \mu \mathrm{s}$, providing a sound basis for correlation and timing analyses.

\section{DATA ANALYSIS}

\subsection{Gamma-ray Event Selection}

Prior to performing a correlation search between the gammaray and radio data sets, the VERITAS data were passed through an analysis pipeline, which reconstructs the arrival direction and the energy of the gamma-ray candidate from the Cherenkov images recorded by the telescopes. For each event, the signal in each PMT is corrected for gain differences between the PMTs and signals which only contain noise are removed. Following this image cleaning, images which have a combined PMT signal (size) above 20 photoelectrons have their second moments calculated about their major and minor axes (Hillas 1985). The arrival direction and impact parameter of the gammaray candidates are then calculated from the intersection points of the major axes of the images from multiple telescopes when projected on the sky and ground planes respectively (Hofmann et al. 1999). Background suppression, including cosmic-ray rejection, is performed by comparing measured event parameters with Monte Carlo gamma-ray simulations. Selection parameters include image brightness and shape, arrival direction, height of shower maximum, and impact distance, and are combined in multi-dimensional energy-dependent look-up tables (Krawczynski et al. 2006). The optimal cut values were chosen a priori by modeling the gamma-ray signal from the Crab pulsar as a power law with an integral flux of $1 \%$ of the Crab Nebula flux above $100 \mathrm{GeV}$ and a spectral index of -4 . A grid search for the optimal gamma-ray cut values was then performed using this simulated signal with real Crab Nebula data as background. The gamma-ray selection parameters and the resulting optimal cut values are the angular separation between the source location and the shower direction, theta $(<0.27)$, mean scaled width $(<1.17)$, mean scaled length $(<1.35)$, and height of shower maximum $(>6.6 \mathrm{~km})$. This cut optimization yielded an energy threshold of $120 \mathrm{GeV}$ for sources with a spectral index of -4 . This gamma-ray analysis is identical to the analysis which was used in our paper on the first detection of the Crab pulsar above $100 \mathrm{GeV}$ (Aliu et al. 2011). The GPS time of the candidate gamma-ray events which passed cuts was converted to barycentric dynamical time and phase-folded using the Crab pulsar monthly timing ephemeris with an in-house software package. The accuracy of these calculations was crosschecked using the Tempo program.

\subsection{Correlation Search Strategy}

The physical mechanisms which are responsible for the emission of GRPs are not well understood. The nature of a possible connection between GRPs and the formation of highenergy incoherent emission from pulsars is also unknown. Given these unknowns, we prepared a correlation search strategy which probed for a connection between GRPs and VHE gamma-ray emission on different timescales, allowing for lagging or leading between gamma-ray and GRP emission. The searches were done by selecting only those gamma-ray events which arrived at the solar system barycenter within a certain time window around a GRP event. The durations of the time windows were specified in units of pulsar rotations. We employed eight different time window durations lasting 1, 3, 9, 27, 81, 243, 729, and 2187 pulsar rotations. This covers time intervals from 0.033 to $72.17 \mathrm{~s}$ in time spacings which scale as log to the base 3. Each time window was used to search for enhanced gamma-ray emission which lagged, led, or was contemporaneous with, the GRP. Thus, for each search window duration, a window was positioned centered on the GRP, with the leading and lagging windows placed directly before and directly after the centered window, respectively (see Figure 2). Finally, searches were performed considering only main pulse GRPs, only interpulse GRPs, and both main and interpulse GRPs combined. Thus, a total of 72 searches were performed.

In each of the 72 searches, the phase of the gamma-ray events which fall within the search window is calculated. In our earlier measurement of pulsed VHE emission from the Crab pulsar (Aliu et al. 2011), we determined the phases of emission to be between -0.013 and 0.009 for the main pulse and between 0.375 and 0.421 for the interpulse. In this study we only consider VERITAS events that fall within these phase regions. When a gamma-ray event falls within the search window defined by a main pulse GRP, it has to lie within the VHE main pulse emission 


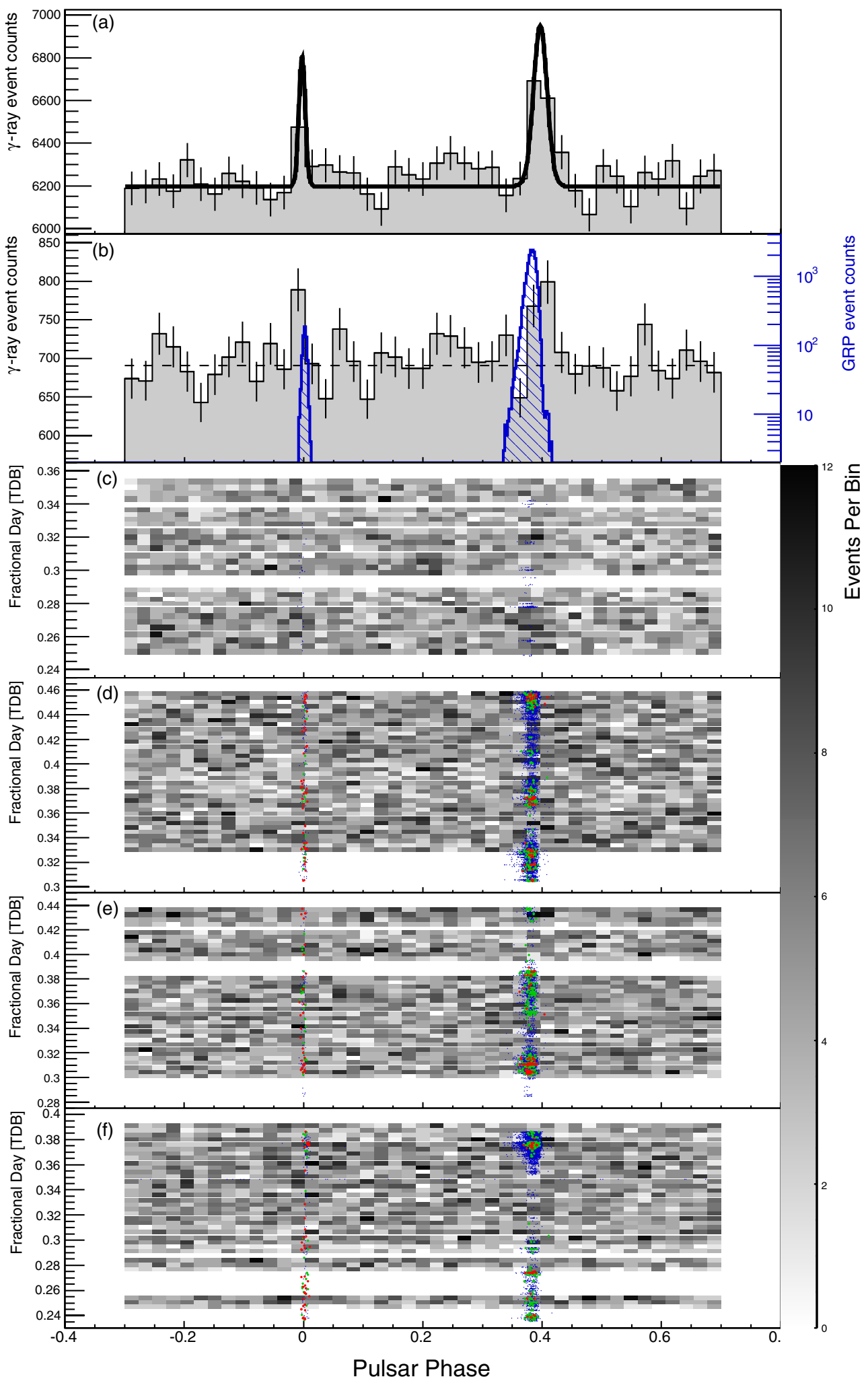

Figure 1. Summary of the gamma-ray and radio observations. Panel (a) shows the gamma-ray pulse profile for the published 107 hr VERITAS Crab Pulsar data set (Aliu et al. 2011). The overlaid solid line is the best-fit function determined from a maximum likelihood fit to the unbinned VERITAS data. Panel (b) shows the gamma-ray (gray) and giant radio pulse (hatching) profiles for the four nights of simultaneous observations. Panels (c)-(f) show the arrival time at the barycenter vs. phase for the observation dates 54829, 55153, 55158, and 55180 in MJD, respectively. The gray-scale histograms show the VERITAS data while the blue, green, and red points are the radio data with energies less than $100 \mathrm{Jy} \mu \mathrm{s}$, greater than $100 \mathrm{Jy} \mu \mathrm{s}$, and greater than $150 \mathrm{Jy} \mu \mathrm{s}$, respectively.

(A color version of this figure is available in the online journal.) 


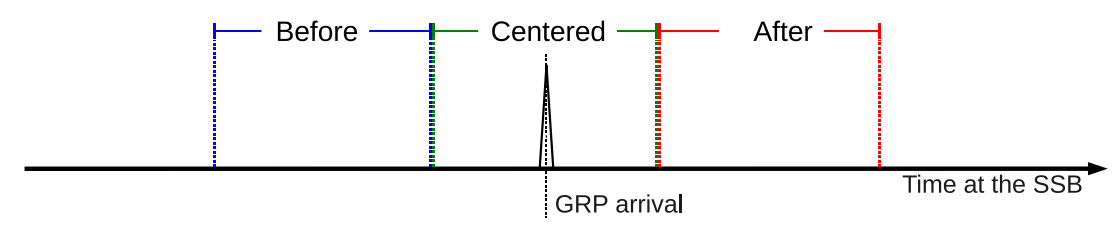

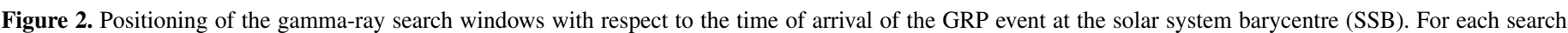

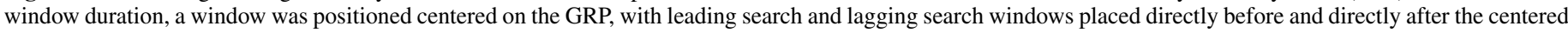
search window, respectively.

(A color version of this figure is available in the online journal.)

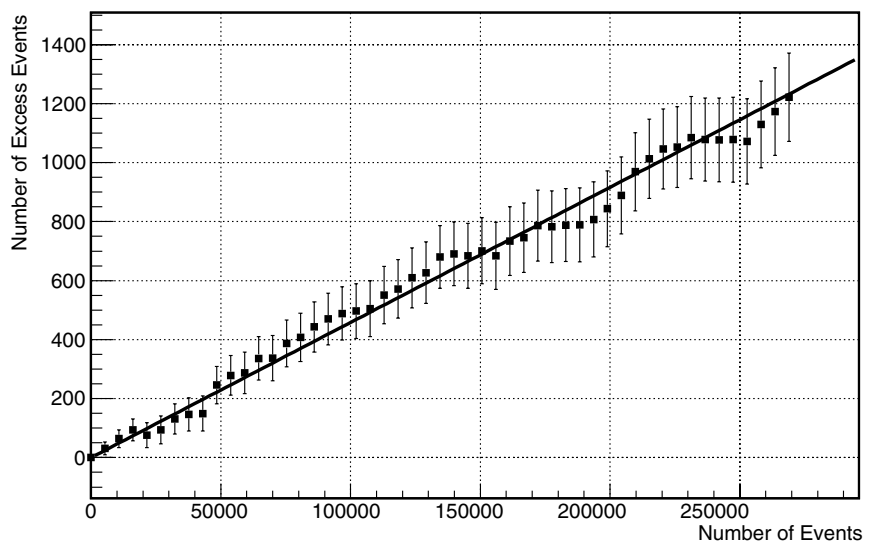

Figure 3. Number of excess gamma-ray events in the phase interval -0.013 to 0.009 (main pulse) and 0.375 to 0.421 (interpulse) plotted against the total number of events for the complete $107 \mathrm{hr}$ VERITAS data set (Aliu et al. 2011). The excess growth is clearly linear. From a total excess of $1256 \pm 130$ in 267,088 events, there are $4.7 \pm 0.48$ excess events in the VHE emission phases per 1000 events selected.

phase range for it to be considered in the enhancement search. Corresponding selection criteria are applied to those gammaray events which fall within a window defined by an interpulse GRP. In the searches which consider both main and interpulse GRPs, gamma-ray events which fall within either of the VHE emission phase ranges are selected. The prescription for these 72 searches was defined before the data sets were analyzed.

\section{MONTE CARLO TIME SERIES}

In order to determine the presence of an enhancement in the VHE emission from the pulsar correlated with GRPs, Monte Carlo time-series data sets were generated to model the gammaray data. Using a bin width of one second, raw trigger rate distributions (number of triggers per second) were compiled for each VERITAS observation run. These distributions were used as probability density functions from which random event times were drawn and sorted, from earliest to latest, producing random time series with the same temporal characteristics as the real VERITAS data. Furthermore, to enable the measurement of the level of any VHE flux increase seen in the VERITAS data, or to facilitate the calculation of flux upper limits, these Monte Carlo time-series data sets were injected with a simulated signal from the Crab pulsar as explained in the following section.

From our earlier analysis of the complete $107 \mathrm{hr}$ VERITAS Crab pulsar data set, we determined that the excess of events, which fall within the VHE main and interpulse emission regions, grows linearly with respect to the total number of events selected (Aliu et al. 2011). This linear growth is plotted in Figure 3. With a total excess of $1256 \pm 130$ in 267,088 events, we determine that there are $4.7 \pm 0.48$ excess events in the VHE emission phases per 1000 events selected. Given this linear
Table 2

Number of Signal and Background Events Which Are Present in the VERITAS Data Recorded on the Four Nights of Observation as Determined by the Linear Relationship Discussed in the Text

\begin{tabular}{lccc}
\hline \hline Date (MJD) & $\begin{array}{c}\text { Total Number } \\
\bar{N}_{T}\end{array}$ & $\begin{array}{c}\text { Number of Excess } \\
\bar{N}_{\text {ex }}\end{array}$ & $\begin{array}{c}\text { Number of Background } \\
\bar{N}_{\text {bg }}\end{array}$ \\
\hline 54829 & 5566 & 26.2 & 5539.8 \\
55153 & 8738 & 41.1 & 8696.9 \\
55158 & 7928 & 37.3 & 7890.7 \\
55180 & 7861 & 37.0 & 7824.0 \\
\hline Total & 30093 & 141.6 & 29951.4 \\
\hline
\end{tabular}

Notes. The number of background events is defined as the total number of events minus the estimated excess. When generating a Monte Carlo time series which models the observations made on a given date, the number of background events to be generated is drawn from a Poisson distribution with a mean $\bar{N}_{\text {bg }}$. The number of pulsar events to be generated is drawn from a Poisson distribution with a mean $\bar{N}_{\text {ex }} \times x_{i}$, where $x_{i}$ is used to scale the number of excess events to the desired pulsar flux level.

relationship, the number of excess events which lie within the VHE emission phases, for any subset of the VERITAS data, can be estimated (see Table 2). Furthermore, we know that the emission peaks in the VERITAS Crab pulsar phasogram can be modeled by two Gaussians sitting on a uniform background. This was determined by an unbinned maximum likelihood fit of the VERITAS phase data (Aliu et al. 2011). Using these two observations we can generate a Monte Carlo time series inhabited by a simulated Crab pulsar signal at any desired flux level.

If a given VERITAS observation has a total number of events, $\bar{N}_{T}$, using the linear relationship discussed above, the number of excess pulsar events expected within this sample, $\bar{N}_{\text {ex }}$, can be estimated. The number of background events is then $\bar{N}_{\text {bg }}=\bar{N}_{T}-\bar{N}_{\text {ex }}$. Now assume we want to model this VERITAS observation with a simulated signal from the Crab pulsar at a flux level $x_{i}$, where $x_{i}$ is in units of the average pulsar flux level measured with VERITAS. We draw a number of background events, $N_{\mathrm{bg}}$, from a Poisson distribution with a mean $\bar{N}_{\text {bg }}$ and we draw a number of pulsar events, $N_{\text {ex }}$, from a Poisson distribution with a mean $\bar{N}_{\text {ex }} \times x_{i}$. Now, using the VERITAS raw data rate distributions, discussed earlier, we draw $N_{T}=N_{\text {bg }}+N_{\text {ex }}$ random arrival times. A fraction of these events, $N_{\text {ex }} / N_{T}$, is randomly selected to contain the injected pulsar signal. This is done by shifting the arrival time of these events to the nearest time which, when barycentered and phase-folded, would correspond to a random phase value drawn from the double Gaussian function which parameterizes the VERITAS Crab pulsar phasogram. This small shift in time $(<17 \mathrm{~ms})$ has no effect on the overall data rate characteristics of the Monte Carlo data sets, and is only applied to a small fraction of randomly selected events within each data set. This procedure was used to 


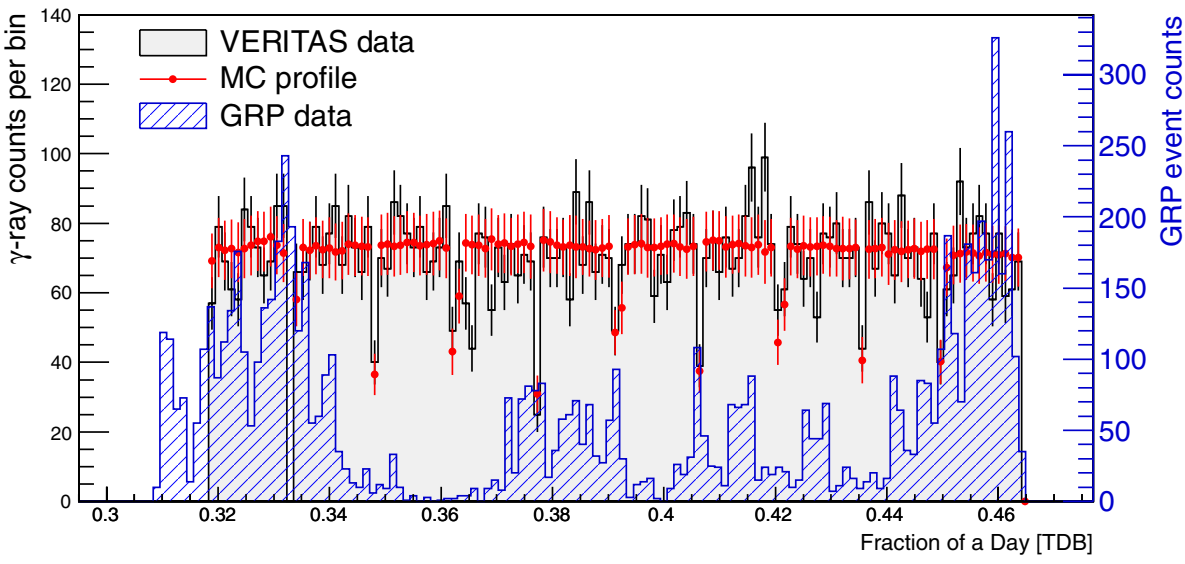

(a) Event Arrival Times

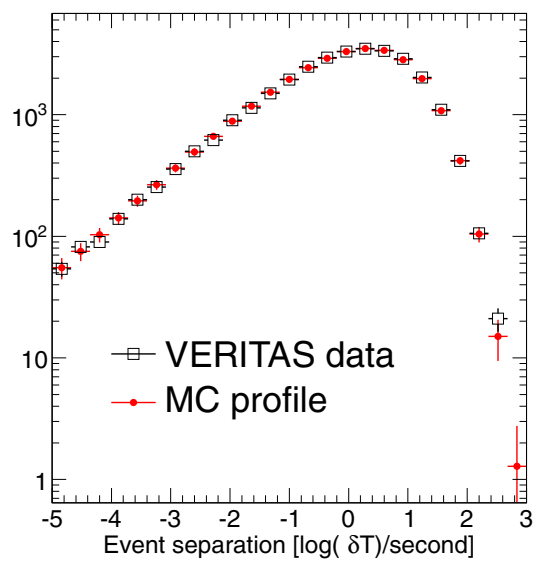

(b) Event Separation

Figure 4. Panel (a) shows the distribution of arrival times at the solar system barycenter of the gamma-ray (gray), radio (hatching), and Monte Carlo data (circles) for the observations made on MJD 55153. The sharp drops in the gamma-ray data rate are due to gaps in observation between successive 20 minute exposures. Panel (b) shows the arrival time event-separation distribution for each pair of consecutive events in the complete gamma-ray and Monte Carlo data sets. In both panels (a) and (b), the Monte Carlo data contain an injected signal from the Crab pulsar at the level of the measured VHE pulsar flux $\left(x_{i}=1\right)$ and are plotted as a profile histogram, with the height and error bar of each bin representing the mean and standard deviation determined from 500 simulated time-series data sets, respectively.

(A color version of this figure is available in the online journal.)

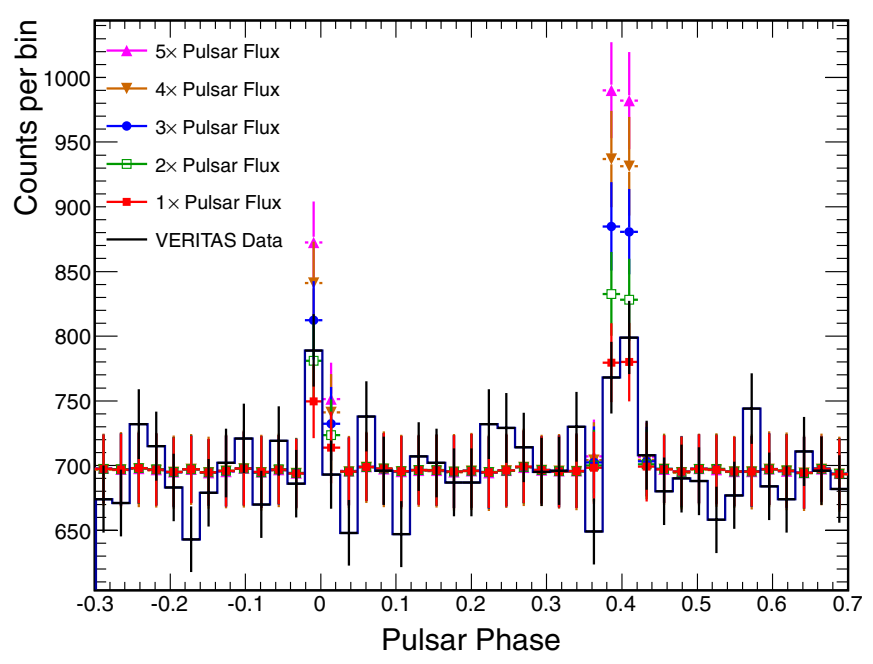

Figure 5. VERITAS Crab pulsar phasogram determined from the $11.6 \mathrm{hr}$ of simultaneous exposure together with phasograms determined from Monte Carlo time-series data sets. The Monte Carlo data contain a simulated signal from the Crab pulsar at various flux levels and are plotted as a profile histogram, with the height and error bar of each bin representing the mean and standard deviation determined from 500 simulated time-series data sets, respectively.

(A color version of this figure is available in the online journal.)

generate sets of Monte Carlo data, containing a signal from the Crab pulsar at a chosen flux level, which model all the data rate characteristics of the real VERITAS data, accounting for the Poisson fluctuations inherent in the VERITAS measurement of the Crab pulsar flux. Examples of the match between the Monte Carlo time-series data sets and the VERITAS gamma-ray data are shown in Figures 4 and 5.

\section{RESULTS}

\subsection{Search for Enhanced Gamma-ray Emission During GRPs}

Each enhancement search yields a number, $N$, which is the number of VERITAS events that meet the specific search criteria, i.e., they fall within a time interval determined by a GRP with a phase value within the required VHE emission

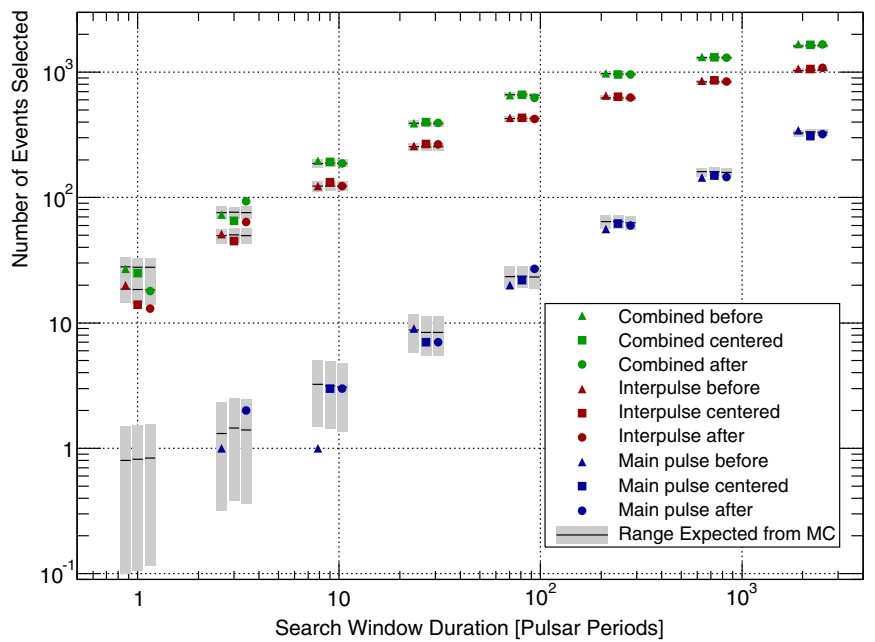

Figure 6. Number of gamma-ray events selected (symbols) vs. the duration of the search window. The gray regions denote $68 \%$ containment intervals about the mean of the distribution (black line) determined from searches performed on the Monte Carlo data. The Monte Carlo data set is composed of 500 simulated time series containing an injected signal from the Crab pulsar at the level of the measured VHE pulsar flux $\left(x_{i}=1\right)$. The absence of a symbol in four of the searches indicates that the number of selected events in each of these cases was zero. All searches return values which are consistent with what is found from searches on the Monte Carlo data sets. The slight negative and positive shift of the $x$-position of the before and after symbols is done as a visual aid to prevent clutter about the common $x$-coordinate value for the before, centered, and after symbols.

(A color version of this figure is available in the online journal.)

phase range. The Monte Carlo time-series data sets were subject to the same search which was performed on the real VERITAS data set. Given that we generated a large number of Monte Carlo time series, a search on a Monte Carlo data set will yield a distribution of the number of selected events, which is approximately Gaussian, and will have a mean, $\mu$, and a variance, $\sigma^{2}=\mu$. The number of events selected in a given search, $N$, can be compared to the mean number expected from the Monte Carlo when no enhancement is present, $\mu$. Such a comparison is plotted in Figure 6, showing the number of 


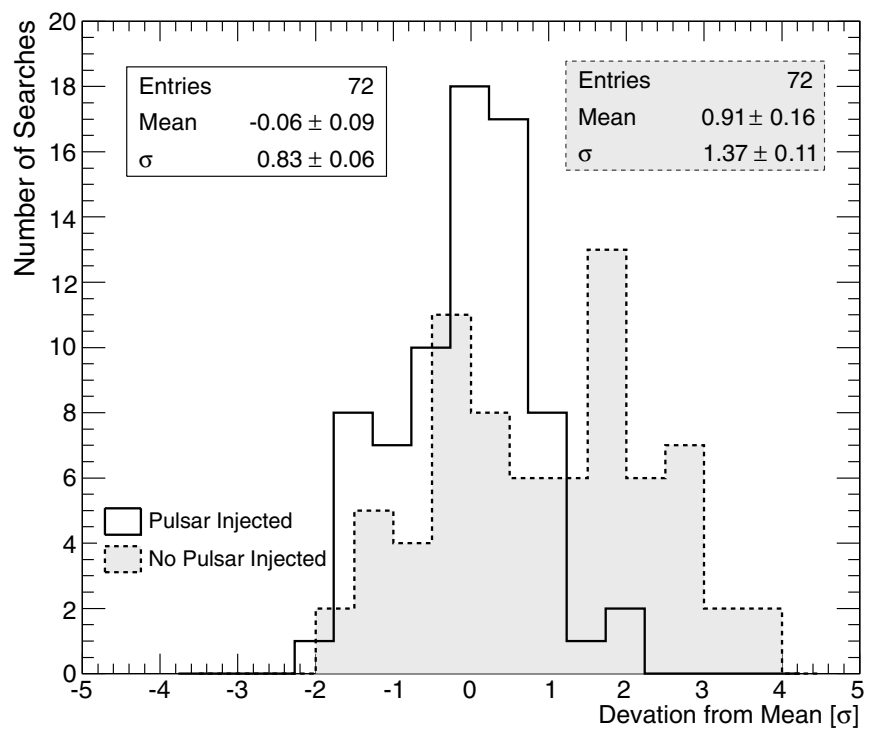

Figure 7. Deviation of number of events selected in the enhancement searches on the real data compared to the mean number expected from the Monte Carlo distributions in units of their standard deviation. The positive shift of the mean of the distribution determined from searches on Monte Carlo data sets with no pulsar signal flux signal injected (gray) is due to presence of the pulsar signal in the VERITAS data set and the absence of a pulsar signal in the Monte Carlo. Including in the Monte Carlo a Crab pulsar signal at the level of the average measured VHE pulsar flux yields a distribution (solid black), which has a mean compatible with zero and a standard deviation close to one. This indicates that there is no significant enhancement in the gamma-ray flux correlated with GRPs. We note that the searches are correlated because the same events can be selected in different searches.

events selected in searches of the VERITAS data, compared to the mean number selected from a Monte Carlo data set. Here, the Monte Carlo data set is composed of 500 simulated time series, each containing an injected signal from the Crab pulsar at the level of the average measured VHE pulsar flux $\left(x_{i}=1\right)$. Using the formula, $S=(N-\mu) / \sigma$, one can determine the statistical significance of any deviation of the measured number of events, from the number expected in the absence of any enhancement. ${ }^{35}$ The significance distribution derived from the 72 searches is plotted in Figure 7. This distribution has a mean compatible with zero and a standard deviation close to one, indicating the absence of any significant enhancement in the VHE gammaray flux within the specified search windows positioned around GRPs observed at $8.9 \mathrm{GHz}$.

\subsection{Calculation of the Upper Limit on the Flux Increase}

We follow the same prescription as Bilous et al. (2011) to compute the upper limit on the VHE flux during the enhancement searches. From Bayes' theorem, the posterior probability that the pulsar flux is $F$ given that we observed $N$ events in an enhancement search is

$$
P(F \mid N)=\frac{P(N \mid F) P(F)}{\int_{0}^{\infty} P\left(N \mid F^{\prime}\right) P\left(F^{\prime}\right) d F^{\prime}},
$$

where $P(N \mid F)$ is the likelihood of selecting $N$ events in a search when the pulsar flux is $F$. For simplicity, all flux values specified

\footnotetext{
35 Twelve of the searches on the Monte Carlo data sets yield distributions which are more Poissonian in shape than Gaussian $(\mu \leqslant 10)$, meaning that the strict equivalence of the significance formula breaks down. The resulting assertions are, however, unchanged.
}

here are cast in units of the average flux of the Crab pulsar measured with VERITAS. $P(F)$, the prior distribution of the flux, is an uninformative prior which we set to be

$$
P(F)= \begin{cases}C & \text { if } 1<=F<=50, \\ 0 & \text { if } F<1, F>50,\end{cases}
$$

where $C$ is a non-zero constant. This means that during the emission of a GRP we consider the pulsar flux to be, with uniform probability, between 1 and 50 times the average Crab pulsar flux, and to have zero probability otherwise.

From the searches performed on the Monte Carlo data sets, we can explore the likelihood value, $P(N \mid F)$, for a range of flux values, by generating a likelihood curve. Each point in the curve is computed by probing the distribution of the number of selected events determined from a Monte Carlo data set generated with a given flux level, $F=x_{i}$ (see Figure 8 for some example distributions). The $y$-value of each point in the curve is the fraction of the Monte Carlo data sets which, when subjected to the specific enhancement search, yielded the same number of coincident events as were found in the real data. In practice, we fit the distribution yielded from searches on the Monte Carlo data set with a Poisson function, and determine the likelihood from the fitted function. This is done to minimize fluctuations caused by the finite statistics used to compile the Monte Carlo distributions. Figure 8 shows some examples of the fits to the Monte Carlo distributions along with the distribution of the reduced $-\chi^{2}$ values $\left(\chi^{2} / \mathrm{ndf}\right)$ for all of the fits performed. From the reduced $-\chi^{2}$ distribution, which has a mean value close to 1 , it is clear that the Poisson functional form accurately describes the distributions derived from searches performed on the Monte Carlo data sets.

The likelihood curve for each search was compiled using Monte Carlo data sets with injected flux levels ranging from $x_{i}=1$ to 50 , with step sizes of 0.1 between 1 and $10,0.25$ between 10 and 20, and 1 between 20 and 50. Given our choice of the prior $P(F)$, and that we evaluated the likelihood at an array of discrete flux levels, Equation (1) for the posterior probability can be rewritten as

$$
P\left(F=x_{i} \mid N\right)=\frac{P\left(N \mid F=x_{i}\right)}{\sum_{i=1}^{160} P\left(N \mid F=x_{i}\right) \Delta x_{i}},
$$

where $i$ runs over the array of simulated flux levels and $\Delta x_{i}$ is step size between each consecutive flux level. This posterior probability curve is normalized and can be integrated yielding a cumulative posterior probability, $P\left(F<x_{\mathrm{ul}} \mid N\right)$, from which one can determine the flux upper limit, $x_{\mathrm{ul}}$, at a chosen confidence level. Figure 9 shows the posterior probability and cumulative distribution functions for three different searches. The flux value where the cumulative probability distribution crosses 0.95 marks the upper limit on the emitted flux correlated with GRPs at the $95 \%$ confidence level.

The upper limits on the flux correlated with GRPs at the 95\% confidence level are plotted in Figure 10 for 64 of the 72 searches. On the shortest timescales probed, the duration of one pulsar period, a limit of $\sim 5-10$ times the average Crab pulsar flux is set on the interpulse and the combined interpulse and main pulse searches. For eight of the searches performed around main pulse GRPs, the posterior probability curve had not converged to zero before a flux value of 50 times the measured flux. This is due to the low rate of main pulse GRPs detected at $8.9 \mathrm{GHz}$, which resulted in a small number of selected gamma-ray events in some of the main pulse GRP searches. The relatively large 

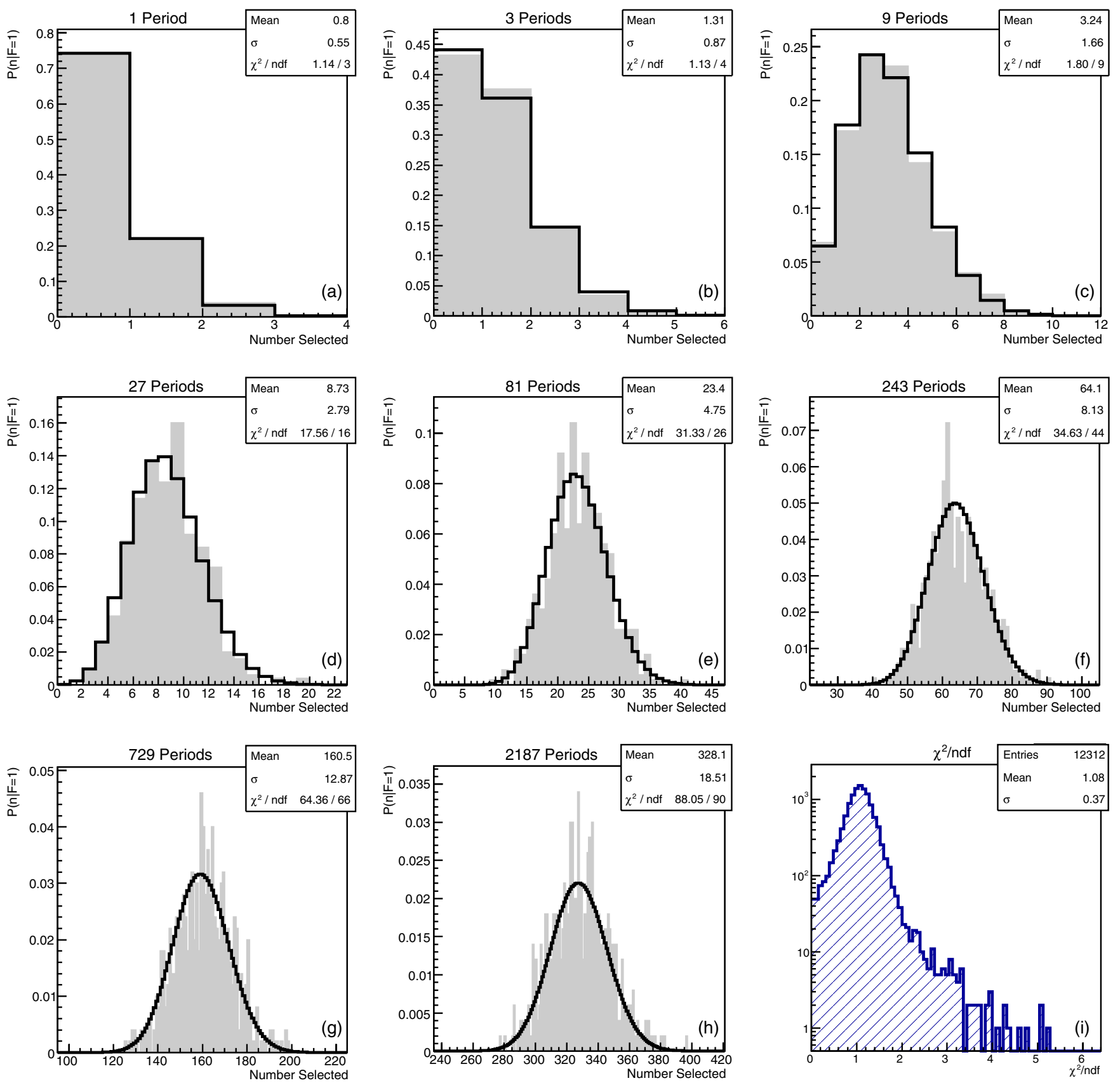

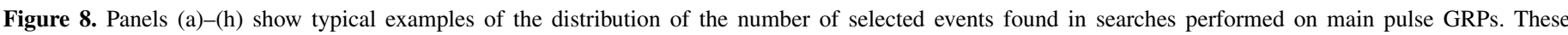

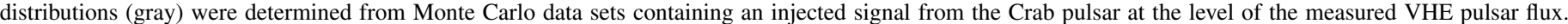

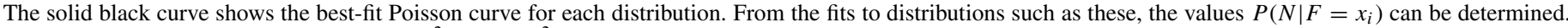

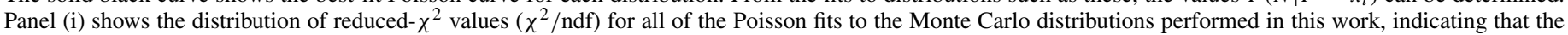
Poissonian form accurately describes the data.

(A color version of this figure is available in the online journal.)

fluctuations inherent in small-number statistics result in wide posterior probability curves and, thus, very high upper-limit values. We do not quote a $95 \%$ confidence level upper-limit value for these searches, but note that it is likely around 50 times the average gamma-ray flux. As the search window size is increased, and thus the statistical sample increases, these fluctuations decrease and the upper-limit values become more constraining. Once enough of the gamma-ray sample is selected due to the increasing size of the search windows, the upper-limit values level out at $\sim 2-2.5$ times the average Crab pulsar flux. We note that for the longest two search windows, 729 and 2187 periods, between $70 \%$ and $80 \%$ of the VERITAS events are selected in the interpulse and combined searches resulting in a reduced enhancement sensitivity in these searches. ${ }^{36}$

\subsection{Estimation of Uncertainty}

As stated earlier, the fraction of Monte Carlo searches which yield the same number of correlated events as were found in

\footnotetext{
36 The $70 \%$ and $80 \%$ values were determined without inclusion of the data acquired on the first day of observations where our GRP count was relatively
} low. 

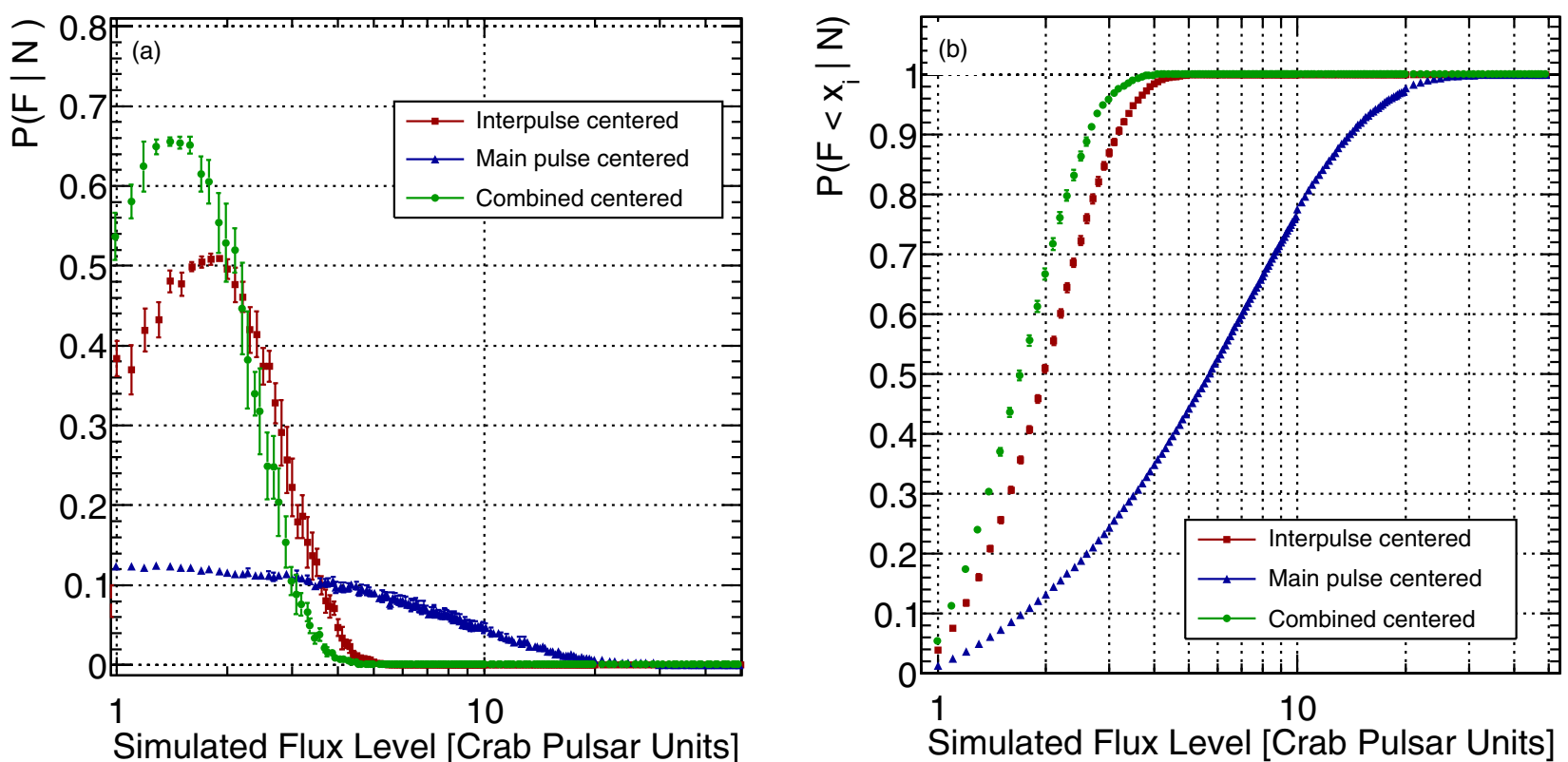

Figure 9. Panels (a) and (b) show the results of correlation searches centered on GRPs with search windows lasting 27 pulsar rotations. Panel (a) plots the posterior probability $P\left(F=x_{i} \mid N\right)$ for a range of flux levels, given the number of events selected in the enhancement search. These probability distributions peak close to a flux value of 1 , meaning that the flux is most likely unchanged during the GRP events. The error bars represent the uncertainty due to finite statistics in the Monte Carlo distributions and the fitting procedure used to determine the probability value from the these distributions. Panel (b) shows $P\left(F<x_{i} \mid N\right)$, the cumulative distributions of the corresponding probability distributions shown in panel (a). From such curves, an upper limit on the level of the enhanced flux can be read at a desired confidence level. In the given example, the 95\% confidence level upper limits on the enhanced flux are 17.2, 3.5, and 2.9 times the flux measured by VERITAS for the main pulse, interpulse, and combined searches, respectively.

(A color version of this figure is available in the online journal.)

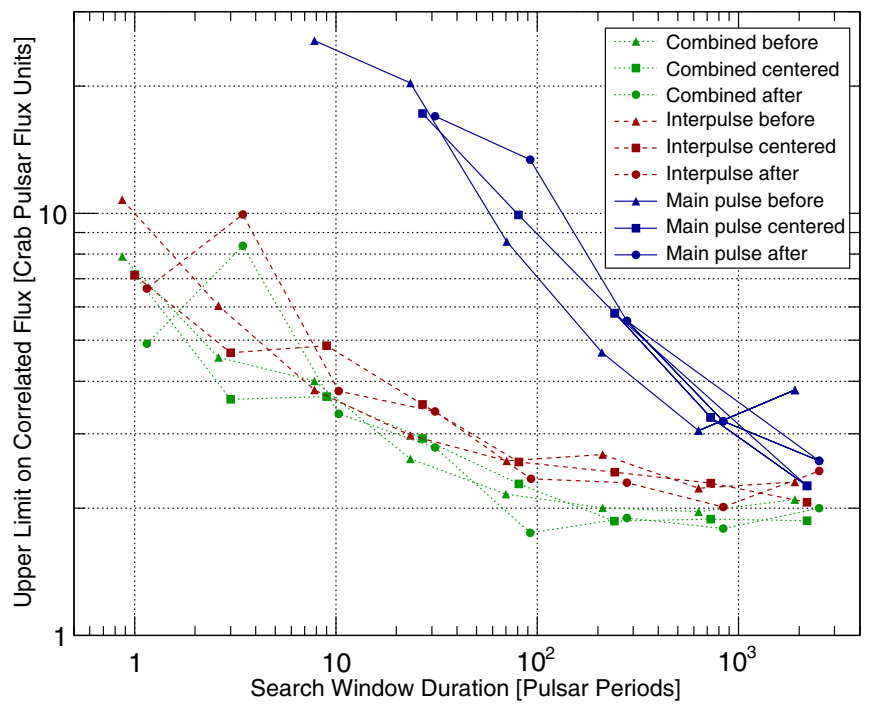

Figure 10. 95\% confidence level upper limit on the gamma-ray flux correlated with GRPs for 64 of the 72 searches. We do not quote an upper-limit value for eight searches with short windows placed around main pulse GRPs, but note that it is likely around or above 50 Crab pulsar flux units. The slight negative and positive shift of the $x$-position of the before and after symbols is done as a visual aid to prevent clutter about the common $x$-coordinate value for the before, centered, and after symbols.

(A color version of this figure is available in the online journal.)

the data, $P(N \mid F)$, was determined from a Poisson fit to the distributions, rather than from the distributions themselves. By adopting this procedure we found that fluctuations in the posterior probability density curves are dramatically reduced, while having little effect on the computed $95 \%$ confidence level upper-limit values. We investigated the uncertainty on the

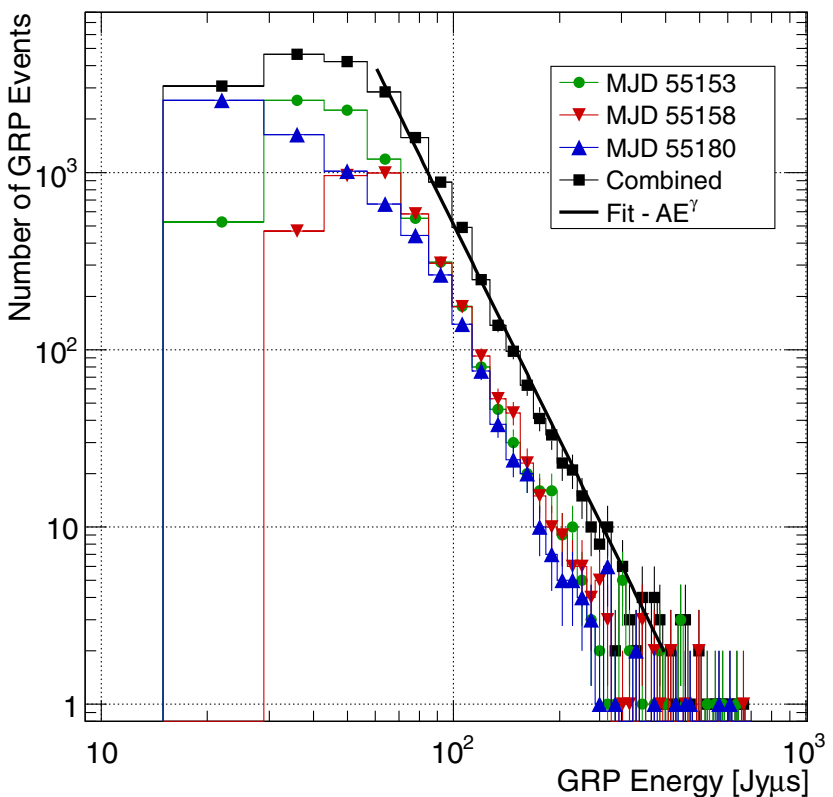

Figure 11. Distribution of giant pulse energies for the latter three nights of observations. The distributions follow a power law, with the combined data set having a spectral index of -4.03 . The different number of GRPs selected below the $\sim 60 \mathrm{Jy} \mu$ s roll-off on each night of observation is due to the different amount of RISS contributions to the GRP flux densities on a given night.

(A color version of this figure is available in the online journal.)

posterior probability values due to the finite statistics in the Monte Carlo distributions and the Poisson fitting procedure.

The $\chi^{2}$ values were determined between a given Monte Carlo distribution and Poisson curves with a range of mean values centered on the best-fit Poisson mean value. From these $\chi^{2}$ values, a fit probability versus Poisson mean curve was 


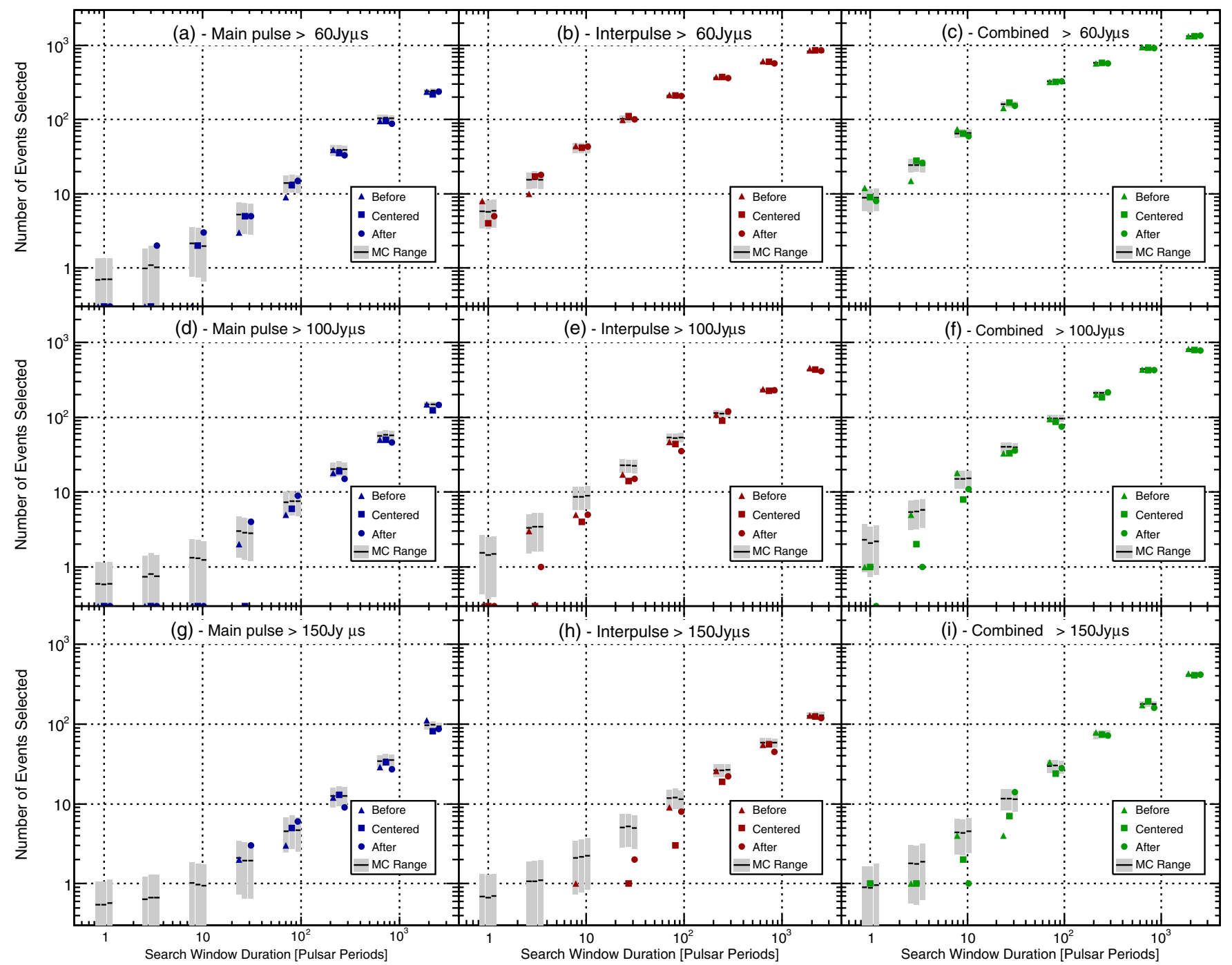

Figure 12. Number of gamma-ray events selected (symbols) vs. the duration of the search window. The gray regions denote $68 \%$ containment intervals about the mean of the distribution (black line) determined from the identical searches performed on Monte Carlo data sets containing an injected signal from the Crab pulsar at the level of the measured VHE pulsar flux. The upper, middle, and lower rows show the results of the enhancement search when restricting the energy of the GRP events to be above 60,100, and $150 \mathrm{Jy} \mu \mathrm{s}$, respectively. No excess (or deficit) is found in any search with a probability equivalent of $3 \sigma$ or higher. See the caption of Figure 6 for a further description of this figure.

(A color version of this figure is available in the online journal.)

generated, and from this curve, random values were drawn. A histogram of $P(N \mid F)$ values was then compiled using Poisson curves with these random values as their mean. The standard deviation of this histogram was then used as the uncertainty on the $P(N \mid F)$ value determined from the best-fit Poisson curve. The uncertainty on the probability values determined in this way is less than $15 \%$ for the bulk of distributions but is occasionally as large as $30 \%$. The error bars on the probability values plotted in Figure 9 were calculated in this way. Other methods to calculate the uncertainty were investigated and found, in general, to yield a smaller uncertainty value. Folding the uncertainty on the probability values into the cumulative distribution, however, the uncertainly on the $95 \%$ confidence level upper-limit value was found to be less than 3\% for the 64 searches which yielded a limiting value. This underlines the robustness of the procedure we used to calculate the upper-limit values.

\subsection{Selecting Only the Most Energetic GRP Events}

Having investigated GRPs whose peak flux density is greater than $7 \sigma$ above the averaged radio signal and observed no VHE enhancement, we now consider only the most energetic GRP events. Due to the low rate of GRP events in the 2008 data set, the following analysis uses the data collected in 2009 only.

Earlier, we estimated that the system equivalent flux density for the 2009 observations was $0.7 \mathrm{Jy}$. The sampling interval for these observations was $3.2 \mu \mathrm{s}$. Thus, the energy of a GRP is

$$
\mathrm{GRP}_{\mathrm{E}}=\frac{\mathrm{GRP}_{\mathrm{S} / \mathrm{N}}}{\sqrt{N_{\mathrm{samp}}}}(0.7 \mathrm{Jy}) \times\left(N_{\mathrm{samp}}\right)(3.2 \mu \mathrm{s}),
$$

where $\mathrm{GRP}_{\mathrm{S} / \mathrm{N}}$ is the signal-to-noise ratio of the GRP and $N_{\text {samp }}$ is the width of the GRP in samples (see Bilous et al. 2012 for more details regarding the energy calculation of GRPs). The effective width of each GRP is determined from the width of the boxcar function which, when convolved with the radio time series, returns the largest $\mathrm{S} / \mathrm{N}$ value for a given pulse. The widest GRP found had a width of 14 samples or $44.8 \mu \mathrm{s}$.

In order to have a common energy scale for observations taken on different nights, we must correct for the effects of RISS. This was done by compiling average radio pulse profiles 
on 80 minute timescales, which is the characteristic timescale of RISS for the Crab at $8.9 \mathrm{GHz}$. The profile for the second half of the observing session on MJD 55180 was the strongest and we chose it as reference pulse profile to which the other profiles were compared. Ratios of the peak $\mathrm{S} / \mathrm{N}$ values were computed between the reference profile and the other profiles and used as RISS correction coefficients to scale the GRP energy values computed by Equation (4). Figure 11 shows the resulting distributions of GRP energy, which follow the expected powerlaw shape with a spectral index of -4.03 . The roll-off below $\sim 60 \mathrm{Jy} \mu \mathrm{s}$ is caused by a bias in our GRP selection, which was based on peak fluxes and reduces the number of broad weak pulses selected. The different number of GRPs selected below the roll-off on each night of observation is due to the different amount of RISS contributions to the GRP flux densities on a given night.

Having computed an RISS-corrected energy value for every GRP event in the 2009 data set, we repeated the correlation search with the VERITAS data. We applied three different energy cuts to the radio events, namely 60, 100, and $150 \mathrm{Jy} \mu \mathrm{s}$. The $60 \mathrm{Jy} \mu$ s threshold was chosen as a low common energy threshold which provides an unbiased sample of GRPs, independent of both the GRP search bias and the RISS conditions during a particular observing session. The other two thresholds were chosen to be "high" and "very high," selecting the most energetic $\sim 6 \%$ and $\sim 1 \%$ of GRPs, respectively. The results of these searches are plotted in Figure 12. We find no significant enhancement $(>3 \sigma)$ in the VHE emission correlated with these energetic GRP events.

\section{DISCUSSION AND CONCLUSION}

Following our study of simultaneous radio and gammaray data, we observe no significant enhancement in VHE gamma-ray emission from the Crab pulsar correlated with GRPs observed at $8.9 \mathrm{GHz}$. Our findings are similar to those previously reported at lower gamma-ray and X-ray energies (Lundgren et al. 1995; Bilous et al. 2011, 2012). Given the level of uncertainty in theories of GRP emission, it is hard to draw firm conclusions resulting from the lack of any observed correlation with VHE emission. We are not aware of any theory with quantitative predictions of correlated emission between GRPs and VHE emission. GRP emission mechanisms associated with changes in plasma coherence will not cause enhancements in incoherent emission. Small and localized changes in the pair-creation rate, which can explain the small (3\%) optical enhancements previously measured by Shearer et al. (2003), would yield VHE flux enhancements which are below our sensitivity.

Enhanced gamma-ray emission in connection with highfrequency interpulse GRPs, as postulated by Lyutikov (2007), is not observed. In this model the primary beam, which generates coherent GRPs through anomalous cyclotron resonance, also emits curvature gamma rays with energies in the tens of $\mathrm{GeV}$ range. It has since been realized that there is a solid upper limit on the energy of curvature radiation in the $\mathrm{Crab}, E_{\gamma} \leqslant 150 \mathrm{GeV}$ (Lyutikov et al. 2012). This is due to the equivalence of the rate of energy loss and rate of acceleration gains achieved by charged particles energized in the outer magnetosphere. Thus, in the Crab pulsar, the curvature photons from the primary beam generally do not reach the VHE energy band. This means that the non-detection of a VHE enhancement, presented here, does not contradict this GRP emission model.
However, it is possible that there is an indirect link between VHE and radio emission for interpulse GRPs. Recent models for the pulsed VHE gamma rays detected from the Crab suggest that inverse Compton emission dominates curvature emission at energies above a few $\mathrm{GeV}$ in the outer magnetosphere (Lyutikov et al. 2012; Du et al. 2012). A recent study of the Geminga pulsar also supports this scenario (Lyutikov 2012). In these models, the primary particle beam is accelerated in a modest electric field (of few percent of the magnetic field strength) in the outer magnetosphere and produces curvature emission up to $\sim 10 \mathrm{GeV}$. Photons in the VHE band are generated by secondary pairs which upscatter their own cyclotron or synchrotron emission. Within the framework of the high-frequency interpulse GRP emission model of Lyutikov (2007), a VHE-GRP connection is thus still expected. In this model, roughly half of the energy of the plasma beam, energized in the magnetic reconnection event, goes into the production of a secondary plasma which can in turn generate enhanced VHE gamma-ray emission via synchrotron self-Compton scattering. The level of the VHE enhancement is, however, difficult to estimate given the uncertainties and fluctuations in the properties of both the primary and secondary beams. This enhancement is likely below the sensitivity of present VHE instruments.

In the model of pulsed VHE emission from the Crab pulsar of Aharonian et al. (2012), where pulsed X-ray emission originating in the magnetosphere is upscattered to VHE energies in the wind beyond the light cylinder, a GRP-VHE correlation should exist at most at the same level as a GRP-X-ray correlation. Since no GRP-X-ray correlation is seen (Bilous et al. 2012) and the upper limit on the enhanced X-ray flux is $30 \%$, any corresponding VHE enhancement is expected to be below $30 \%$ and thus below our sensitivity.

This research is supported by grants from the U.S. Department of Energy Office of Science, the U.S. National Science Foundation, and the Smithsonian Institution, by NSERC in Canada, by Science Foundation Ireland (SFI 10/RFP/ AST2748), and by STFC in the UK. We acknowledge the excellent work of the technical support staff at the Fred Lawrence Whipple Observatory and at the collaborating institutions in the construction and operation of the instrument. The National Radio Astronomy Observatory is a facility of the National Science Foundation operated under cooperative agreement by Associated Universities, Inc.

\section{REFERENCES}

Abdo, A. A., Ackermann, M., Ajello, M., et al. 2010, ApJ, 708, 1254

Aharonian, F. A., Bogovalov, S. V., \& Khangulyan, D. 2012, Nature, 482, 507

Aleksić, J., Alvarez, E. A., Antonelli, L. A., et al. 2012, A\&A, 540, A69

Aliu, E., Arlen, T., Aune, T., et al. 2011, Science, 334, 69

Argyle, E., Baird, G., Grindlay, J., Helmken, H., \& Omongain, E. 1974, Nuovo Cimento B, 24, 153

Bilous, A. V., Kondratiev, V. I., McLaughlin, M. A., et al. 2011, ApJ, 728, 110

Bilous, A. V., McLaughlin, M. A., Kondratiev, V. I., \& Ransom, S. M. 2012, ApJ, 749,24

Burke-Spolaor, S., Johnston, S., Bailes, M., et al. 2012, MNRAS, 423, 1351

Cordes, J. M., Bhat, N. D. R., Hankins, T. H., McLaughlin, M. A., \& Kern, J. 2004, ApJ, 612, 375

Davies, J. M., \& Cotton, E. S. 1957, Solar Energy, 1, 16

Du, Y. J., Qiao, G. J., \& Wang, W. 2012, ApJ, 748, 84

Fierro, J. M., Michelson, P. F., Nolan, P. L., \& Thompson, D. J. 1998, ApJ, 494, 734

Fomin, V. P., Stepanian, A. A., Lamb, R. C., et al. 1994, Astropart. Phys., 2, 137

Hankins, T. H., \& Eilek, J. A. 2007, ApJ, 670, 693 
Hankins, T. H., Kern, J. S., Weatherall, J. C., \& Eilek, J. A. 2003, Nature, 422, 141

Hillas, A. M. 1985, in 19th International Cosmic Ray Conf. (La Jolla), 3, 445

Hofmann, W., Jung, I., Konopelko, A., et al. 1999, Astropart. Phys., 12, 135

Holder, J., Atkins, R. W., Badran, H. M., et al. 2006, Astropart. Phys., 25, 391

Knight, H. S. 2006, Chin. J. Astron. Astrophys., 6, 41

Krawczynski, H., Carter-Lewis, D. A., Duke, C., et al. 2006, Astropart. Phys., 25,380

Kuiper, L., Hermsen, W., Cusumano, G., et al. 2001, A\&A, 378, 918

Lundgren, S. C., Cordes, J. M., Ulmer, M., et al. 1995, ApJ, 453, 433

Lyne, A. G., Pritchard, R. S., \& Graham-Smith, F. 1993, MNRAS, 265, 1003

Lyutikov, M. 2007, MNRAS, 381, 1190

Lyutikov, M. 2012, ApJ, 757, 88
Lyutikov, M., Otte, N., \& McCann, A. 2012, ApJ, 754, 33

Mineo, T., Ferrigno, C., Foschini, L., et al. 2006, A\&A, 450, 617

Moffett, D. A., \& Hankins, T. H. 1996, ApJ, 468, 779

Oosterbroek, T., Cognard, I., Golden, A., et al. 2008, A\&A, 488, 271

Popov, M. V., Soglasnov, V. A., Kondrat'Ev, V. I., et al. 2006, Astron. Rep., 50,55

Popov, M. V., \& Stappers, B. 2007, A\&A, 470, 1003

Romani, R. W. 1996, ApJ, 470, 469

Shearer, A., Stappers, B., O'Connor, P., et al. 2003, Science, 301, 493

Soglasnov, V. 2007, in Proc. 363. WE-Heraeus Seminar on Neutron Stars and Pulsars: 40 Years After the Discovery, ed. W. Becker \& H. H. Huang (Garching: Max Planck Institut für extraterrestrische Physik), 68

Weekes, T. C., Cawley, M. F., Fegan, D. J., et al. 1989, ApJ, 342, 379 